\title{
Potencialidades das intervenções grupais em Centros de Atenção Psicossocial Álcool e Drogas
}

\author{
Potential of group interventions in Psychosocial Care Centers for Alcohol and Drugs \\ Potencial de las intervenciones grupales en los Centros de Atención Psicosocial por Alcohol y Drogas
}

\author{
Johnatan Martins Sousa ${ }^{1}$ (D) \\ Marciana Gonçalves Farinha² (1) \\ Nathália dos Santos Silva ${ }^{1}$ (D) \\ Camila Cardoso Caixeta $^{1}$ (1) \\ Roselma Lucchese ${ }^{3}$ (1) \\ Elizabeth Esperidião ${ }^{1}$ (D)
}

1. Universidade Federal de Goiás, Faculdade de Enfermagem. Goiânia, GO, Brasil.

2. Universidade Federal de Uberlândia, Instituto de Psicologia. Uberlândia, MG, Brasil.

3. Universidade Federal de Catalão, Departamento de Enfermagem. Catalão, GO, Brasil.
Autor correspondente:

Johnatan Martins Sousa.

Email: johnatanfen.ufg@gmail.com

\section{Recebido em 02/08/2021.}

Aprovado em 11/10/2021.

DOI:https://doi.org/10.1590/2177-9465-EAN-2021-0294

\section{Resumo}

Objetivo: analisar as potencialidades das intervenções grupais em Centros de Atenção Psicossocial Álcool e Drogas na perspectiva dos profissionais. Método: pesquisa-intervenção de abordagem qualitativa. Participaram 30 profissionais de saúde mental de quatro Centros de Atenção Psicossocial Álcool e Drogas da região central do Brasil. Os dados foram coletados por meio de instrumentos autoaplicáveis e rodas de conversa com roteiro semiestruturado. Para a análise dos dados, utilizou-se a Análise de Conteúdo, modalidade Temática, com o auxílio do software ATLAS.ti. Resultados: a categoria temática Potencialidades das intervenções grupais contemplou quatro subcategorias que abordaram aspectos relacionados à estrutura física, aos recursos materiais, aos aspectos dos usuários, aos profissionais e aos processos de trabalho. Considerações finais e as implicações para a prática: a potência da prática com grupos estava presente em grande parte dos atendimentos nos serviços pesquisados com a identificação de inúmeros fatores terapêuticos aos usuários. As intervenções aumentaram a fluidez dos processos de trabalho, razão pela qual necessitam ser mais difundidas e implementadas pelas equipes multidisciplinares nos serviços de saúde mental.

Palavras-chave: Assistência à saúde mental; Equipe de Assistência ao Paciente; Processos grupais; Saúde mental; Usuários de drogas.

\section{Abstract}

Objective: to analyze the potential of group interventions in Psychosocial Care Centers for Alcohol and Drugs from the perspective of professionals. Method: intervention-research of qualitative approach. Thirty mental health professionals from four Psychosocial Care Centers for Alcohol and Drugs in the central region of Brazil participated. The data was collected through self-applied instruments and conversation rounds with a semi-structured script. For data analysis, we used Content Analysis, Thematic modality, with the help of the ATLAS.ti software. Results: the thematic category Potential of group interventions contemplated four subcategories that approached aspects related to the physical structure, material resources, user aspects, professionals and work processes. Final considerations and implications for practice: the power of the practice with groups was present in much of the care in the services researched with the identification of numerous therapeutic factors for users. The interventions increased the fluidity of the work processes, which is why they need to be more widespread and implemented by multidisciplinary teams in mental health services.

Keywords: Mental Health Assistance; Patient Care Team; Group Processes; Mental Health; Drug Users.

\section{Resumen}

Objetivo: analizar el potencial de las intervenciones grupales en los Centros de Atención Psicosocial por Alcohol y Drogas, desde la perspectiva de los profesionales. Método: investigación de intervención con enfoque cualitativo. Participaron treinta profesionales de la salud mental de cuatro Centros de Atención Psicosocial por Alcohol y Drogas de la región central de Brasil. Los datos se recolectaron a través de instrumentos autoadministrados y ruedas de conversación, con un guión semiestructurado. Para el análisis de los datos se utilizó el Análisis de Contenido, modalidad temática, con la ayuda del software ATLAS.ti. Resultados: la categoría temática Potenciales de intervenciones grupales incluyó cuatro subcategorías que abordaron aspectos relacionados con la estructura física, recursos materiales, aspectos de usuarios, profesionales y procesos de trabajo. Consideraciones finales e implicaciones para la práctica: el poder de la práctica con grupos estuvo presente en gran parte de las consultas en los servicios encuestados, identificando numerosos factores terapéuticos para los usuarios. Las intervenciones aumentaron la fluidez de los procesos de trabajo, por lo que necesitan ser más generalizadas e implementadas por equipos multidisciplinares en los servicios de salud mental.

Palabras clave: Atención a la salud mental; Grupo de Atención al Paciente; Procesos grupales; Salud mental; Consumidores de drogas. 


\section{INTRODUÇÃO}

O uso abusivo de drogas acarreta danos crônicos à saúde e aos relacionamentos interpessoais estabelecidos pelo indivíduo, especialmente no âmbito familiar ${ }^{1}$. Tal comportamento, ao apresentar sintomas recorrentes e específicos, pode levar a pessoa a desenvolver transtornos por abuso de substâncias psicoativas $^{2}$.

Nesse sentido, o tratamento da dependência de substâncias psicoativas não deve se restringir à abstinência, mas deve ser centrado na reabilitação psicossocial do sujeito ${ }^{3}$ com a oferta de atendimento aos cuidadores desses indivíduos ${ }^{4}$. Para tanto, a atenção à saúde mental é alicerçada no constructo de desinstitucionalização e modelo psicossocial e requer profissionais capazes de acessar as necessidades dos usuários que buscam o cuidado para a concretização de um tratamento holístico ${ }^{5}$.

Nesse contexto, o modelo de atenção psicossocial proposto pela Reforma Psiquiátrica Brasileira é resultado de um processo histórico de disputa de concepções epistemológicas e simbólicas sobre a loucura e o adoecimento mental, que influenciaram os modelos assistenciais e as práticas de cuidado, tendo a desconstrução do modelo manicomial como premissa. O processo de desinstitucionalização vai muito além da substituição do cuidado hospitalocêntrico por serviços de base comunitária e territorial, envolve o conceito ampliado de saúde e integralidade, nega a violenta prática do encarceramento, busca a inclusão social e a promoção da cidadania e reconhece as pessoas como singulares 6 .

Para evitar o encarceramento e garantir a livre circulação das pessoas com problemas mentais pelos serviços, pela comunidade e pela cidade, a Rede de Atenção Psicossocial (RAPS) foi proposta. É composta por serviços e por métodos opostos e substitutivos ao paradigma manicomial imprescindíveis para provocar processos emancipatórios e desencadeadores de potências de vida ${ }^{6}$. Dentre os serviços que compõem a RAPS, destacam-se os Centros de Atenção Psicossocial (CAPS), que são comunitários e de base territorial para o atendimento de casos complexos de adoecimento mental e/ou uso abusivo de álcool e outras drogas, por meio de Projetos Terapêuticos Singulares (PTS) ${ }^{7}$.

Por isso, a modalidade de CAPS é responsável pelo direcionamento à assistência exclusiva para os usuários abusivos de drogas e o Centro de Atenção Psicossocial Álcool e Drogas (CAPSad) oferta inúmeras estratégias de cuidado ${ }^{8}$, incluindo atendimentos grupais ${ }^{9}$. Assim, uma importante ferramenta de acolhimento e de tratamento é a experiência de escuta, a qual pode ser individual ou grupal ${ }^{10,11}$. Dentre todas as estratégias de cuidado disponibilizadas pelos CAPS, as práticas grupais são as de interesse desta investigação por seu evidente potencial terapêutico ${ }^{12}$.

Efetivamente, as intervenções terapêuticas grupais são estratégias de cuidado em destaque no campo da atenção psicossocial, voltada para o acompanhamento de pessoas e para a assistência direta aos usuários que apresentam prejuízos relacionados ao uso abusivo de álcool e outras drogas. Do mesmo modo, para os familiares desses sujeitos, que passam por um processo de sofrimento e adoecimento mental devido às repercussões deste envolvimento com drogas.

Nessa perspectiva, a utilização do grupo como ferramenta de cuidado teve início no século XXe, com o decorrer do tempo, pesquisas no campo da dinâmica grupal têm contribuído com a socialização dos seus benefícios na área da saúde ${ }^{2}$. Assim, a tecnologia grupal pode ser compreendida como a união de fundamentação teórica, metodológica e tecnológica utilizada em diversos cenários da atenção à saúde, educação e investigação científica $^{13}$.

Além disso, a caraterística de um grupo é definida pela união de duas ou mais pessoas que interagem para o alcance de objetivos em comum, mediados pela realização de uma tarefa, e, deste processo interativo, podem emergir diversos fenômenos ${ }^{14}$. Dessa forma, nos grupos terapêuticos no cenário da saúde mental, os relacionamentos entre os seus integrantes são construídos por meio de momentos de escuta, socialização de experiências de vida, reflexão e empatia ${ }^{15}$. Portanto, a intervenção grupal é uma potente modalidade de cuidado que pode ser utilizada no tratamento de pessoas com transtornos mentais ${ }^{16}$.

Nos CAPS, as intervenções grupais podem proporcionar fluidez nos processos de trabalho e estimular ações multiprofissionais coletivas $^{10}$, que favorecem, aos usuários dos dispositivos de saúde, a exploração e o trabalho de sentimentos, desenvolvem processos criativos de enfrentamento de problemas e proporcionam tranquilidade e relaxamento ${ }^{17}$. Nesses serviços, há uma diversidade de atividades e de processos grupais, porém, ainda pouco estudados na perspectiva terapêutica ${ }^{18}$.

Portanto, esta investigação justifica-se diante do cenário controverso de saúde mental no Brasil, com a retomada persistente da ideia da supervalorização de práticas tradicionais de diagnóstico e tratamento medicamentoso, em detrimento das outras formas de cuidado, que valorizam a escuta e a palavra, a educação em saúde, o apoio psicossocial e o vínculo ${ }^{19}$. E, ainda, pela necessidade de investimentos em projetos, serviços e ações que promovam e superem os desafios do modelo psicossocial, o cuidado em liberdade das pessoas com sofrimento psíquico grave e transtornos relacionados ao uso problemático de drogas ${ }^{6,20}$.

Nessa direção, é importante a implementação de pesquisas científicas que tratem da sistematização da assistência à saúde mental dos usuários assistidos nos CAPS ${ }^{21}$, para a contribuição e reflexões para o fortalecimento da RAPS no cenário brasileiro ${ }^{4}$, pois, apesar de o grupo ser uma ferramenta de cuidado que proporciona o protagonismo do usuário durante o processo assistencial em saúde mental, ainda há uma escassez de orientações para a operacionalização do trabalho com grupos de forma assertiva pelas equipes de assistência à saúde dos CAPS nos documentos oficiais ${ }^{22}$, o que compromete a avaliação dos benefícios dessa modalidade de atendimento no cenário da atenção psicossocial.

Por essa motivação, são imperiosas a realização e a divulgação de estudos que tratam dos benefícios, da aplicação e assertividade da assistência terapêutica ofertada pelas equipes 
dos CAPS. Entende-se que as evidências científicas a respeito do exercício de práticas qualificadas, como é o caso de intervenções grupais em CAPS, são ferramentas poderosas para responder aos ataques infundados a despeito da ineficiência do cuidado ofertado por esses serviços. Ao partir deste cenário, o objetivo deste estudo foi analisar as potencialidades das intervenções grupais em Centros de Atenção Psicossocial Álcool e Drogas na perspectiva dos profissionais.

\section{MÉTODO}

Pesquisa de abordagem qualitativa, fundamentada no referencial da pesquisa-intervenção, que é caracterizada por uma construção conjunta e interativa entre todos os atores envolvidos e associa, durante o percurso da investigação, uma intervenção que possibilita o processo analítico dos fenômenos ${ }^{23}$. O estudo atendeu aos critérios consolidados para a divulgação de estudos qualitativos (COREQ) ${ }^{24}$.

A pesquisa foi desenvolvida em quatro CAPSad situados em um município do Estado de Goiás (GO), Brasil, sendo dois serviços classificados como do tipo II, um do tipo III e um infantojuvenil, cuja classificação varia em termos do tamanho da população, turno de trabalho e faixa etária dos usuários. No momento da coleta de dados, ao todo, 87 profissionais estavam incluídos nas equipes e 30 deles participaram do estudo, considerando-se os critérios: ser técnico de um dos serviços com idade superior a 18 anos; profissional com experiência ou estar operacionalizando grupos no momento da investigação. Excluíram-se os profissionais que trabalhavam estritamente na administração e limpeza dos serviços e os que estavam afastados do CAPSad por motivo de licença ou férias.

Em relação à experiência com coordenação de grupos, 25 dos 30 profissionais estavam atuando como facilitadores de atendimentos grupais no momento da coleta de dados e, destes, 21 assinalaram experiência nessa modalidade de cuidado por mais de seis meses e quatro por um período inferior a este no contexto dos CAPSad. Apesar dos demais coordenadores não estarem trabalhando com intervenções grupais no momento da coleta de dados, na etapa do acolhimento e sensibilização dos profissionais nas rodas de conversa, afirmaram possuir experiências anteriores na condução de grupos.

Outra estratégia pertinente foi a realização de um teste piloto que verificou as técnicas utilizadas para a coleta de dados pelo CAPS infantojuvenil estadual pela equipe de pesquisadores. Vale salientar que os participantes desse teste não foram incluídos no estudo e os dados oriundos dessa etapa foram analisados com o intuito de verificar se os recursos utilizados atenderiam ao caminho metodológico proposto. Após os ajustes necessários, os encontros para a coleta de dados foram agendados junto aos gestores das instituições participantes, os quais ocorreram nos dias das reuniões de equipe dos respectivos serviços.

Após as estratégias citadas, houve a coleta de dados entre os meses de março e abril de 2019, consistindo em três momentos: aplicação do questionário de caracterização sociodemográfica e profissiográfica e do instrumento para a identificação de Fatores
Terapêuticos (FT) do grupo de acordo com o referencial de Yalom e Leszcz ${ }^{25}$, realização de rodas de conversa e de uma oficina educativa.

Assim, a pesquisa de campo foi conduzida por três pesquisadores, sendo um pós-graduado em dinâmica de grupo e gestão de equipes, uma mestra com formação em consultoria e gestão de grupos e um auxiliar de pesquisa graduando em Enfermagem. Um dos pesquisadores já tinha proximidade com profissionais de dois dos serviços incluídos no estudo pelo fato de ter realizado estágio supervisionado nas instituições e outra pesquisadora era apoiadora institucional dos serviços incluídos no estudo e integrante da Gerência de Saúde Mental do Estado onde a pesquisa foi implementada.

Após os participantes responderem aos instrumentos autoaplicáveis, foram realizadas quatro rodas de conversa, uma em cada CAPSad, com duração aproximada de duas horas, sendo cada uma sistematizada em três etapas: a acolhida dos profissionais pelos facilitadores, objetivando manter um clima de trocas e diálogo entre eles; a exploração da temática subsidiada pela questão de discussão e pela sistematização do processo vivenciado naquele momento e, por último, a avaliação por meio da legitimação do produto gerado pelos membros participantes ${ }^{26}$. A principal questão para a discussão foi: Vocês identificam, nos seus atendimentos grupais, fatores que facilitam e fatores que dificultam a condução dos grupos?

Na etapa da exploração, no momento da problematização do tema, participaram 29 profissionais, quatro no CAPSad 1, nove no CAPSad 2, oito no CAPSad 3 e oito no CAPSad 4, pois uma das profissionais teve que se ausentar para prestar assistência.

Com efeito, a roda de conversa, utilizada como método de coleta de dados, também teve o caráter de uma intervenção, ainda que pontual, na medida em que, ao explorar o tema, em um ambiente propício para a fala, a escuta e a reflexão, gerou um processo interpessoal de discussão, favorecendo a tomada de consciência da potência das ações grupais que os profissionais estavam empreendendo nos seus respectivos serviços. Por fim, os encontros foram gravados em áudio, tendo sido ainda registrados por um dos pesquisadores que se posicionou como observador participante do processo.

Com a última etapa da coleta de dados e com base no material analisado, surgiu a ideia de um planejamento de uma oficina educativa, visando a validar os dados produzidos nas rodas de conversa junto aos participantes e atender aos seus questionamentos em relação às práticas grupais no sentido de melhor instrumentalizá-los para o trabalho. Esta atividade ocorreu em dois grupos, prevendo fomentar as discussões.

Ao longo de toda a investigação, foram feitos registros em diário de campo pela equipe de pesquisadores. Os dados coletados nas rodas de conversa foram submetidos à Análise de Conteúdo, modalidade Temática ${ }^{27}$, com o auxílio do software ATLAS.ti na construção do corpus do estudo. As três fases de análise foram rigorosamente seguidas, desde as exaustivas leitura e seleção dos dados que passariam pelo processo analítico, seguidas da exploração do material, em que foi realizada a codificação das 
unidades de registro e contexto para o agrupamento das famílias e construção das categorias temáticas e de subcategorias. Em suma, o tratamento dos resultados foi obtido com a elaboração de figuras, quadros e diagramas com vistas à apresentação das informações coletadas.

O estudo obteve a aprovação do Comitê de Ética em Pesquisa (CEP) sob o Protocolo inicial no 821.767 e Protocolo final $n .{ }^{\circ}$ 3.951.500. Os profissionais assinaram o Termo de Consentimento Livre e Esclarecido (TCLE) em conformidade com as recomendações da Resolução $n^{\circ} 466$, de $2012^{28}$. Para preservar o anonimato, os participantes foram codificados com a letra $\mathrm{P}$ seguida do número de ordem de exposição verbal nas rodas de conversa de acordo com os CAPS aos quais estão vinculados, também enumerados de um a quatro.

\section{RESULTADOS}

A maioria dos participantes foi composta por profissionais do sexo feminino com uma diversidade de categorias profissionais e com idades variando entre 28 e 64 anos. A Tabela 1 mostra os dados sociodemográficos e de caracterização profissional dos colaboradores em frequência absoluta.

A análise de conteúdo levou à constituição da categoria temática Potencialidades das intervenções grupais, que se

Tabela 1. Características sociodemográficas e profissiográficas dos participantes do estudo. Goiânia/GO, Brasil, 2019.

\begin{tabular}{|lc|}
\hline \multicolumn{1}{|c}{ Variáveis } & Profissionais $(\mathrm{N}=30)$ \\
\hline Idade & 1 \\
\hline 20 a 29 anos & 10 \\
\hline 30 a 39 anos & 5 \\
\hline 40 a 49 anos & 10 \\
\hline 50 a 59 anos & 4 \\
\hline$>60$ anos & \\
\hline Sexo & 3 \\
\hline Masculino & 27 \\
\hline Feminino & \\
\hline Formação profissional & 2 \\
\hline Enfermagem & 3 \\
\hline Técnico em Enfermagem & 10 \\
\hline Psicologia & 5 \\
\hline Serviço Social & 3 \\
\hline Musicoterapia & 2 \\
\hline Terapia Ocupacional & 1 \\
\hline Educação Física & 3 \\
\hline Artes & 1 \\
\hline Gestão Pública & \\
\hline
\end{tabular}

Fonte: elaborado pelos autores. desdobrou em quatro subcategorias: estrutura física e recursos materiais; usuários; profissionais e processos de trabalho dos CAPSad, como ilustra a Figura 1.

A subcategoria Potencialidades das intervenções grupais relacionadas à estrutura física e aos recursos materiais remete à percepção dos profissionais sobre os elementos positivos ligados aos aspectos estruturais e instrumentais dos serviços que favorecem as intervenções como a realização de grupos fora do CAPS. Eles disseram:

Tem o grupo de caminhada, nós atravessamos aqui na praça, na paróquia, tem uma calçada grande em volta, dá um percurso bom para caminhar. (P29 - CAPS 4)

Os participantes relataram que o espaço é adequado para a realização das intervenções e as salas tinham boa iluminação como potencialidades para a prática com grupos:

A sala tem uma boa iluminação para trabalhar, faz uma mesclagem entre a questão terapêutica e um pouco de técnica. Então, como a gente está trabalhando com desenho, você precisa ter uma boa iluminação, essa sala tem! (P1 - CAPS 1)

[...] a estrutura foi construída para o CAPS, é uma sede própria, diferente da maioria dos CAPS que ficam em casas alugadas, aqui, foi construído para ser um CAPS 3. Então, acaba que as estruturas das oficinas é uma das melhores que tem em termos de espaço físico [...]. (P23 - CAPS 4)

A subcategoria Potencialidades das intervenções grupais relacionadas aos aspectos dos usuários emergiu da observação dos profissionais sobre os fenômenos que permeiam os membros dos grupos implementados por eles e que contribuem para a fluidez dessa modalidade de cuidado como a adesão dos usuários à proposta dos grupos:

[...] tem a participação do usuário que é boa aqui no CAPS, toda atividade que a gente propõe, a gente tem uma boa adesão, sim, a gente propõe uma atividade, logo, já está cheio o grupo. (P25 - CAPS 4)

Os participantes também verbalizaram que o compartilhamento de experiências, a construção de vínculos entre os integrantes do grupo e a conscientização de mudança de comportamento por meio dessas intervenções também favorecem essa prática nos CAPSad:

[...] a experiência do outro ajuda para o fortalecimento de quem está mais fragilizado, a experiência do outro é uma ajuda mútua, a partilha que é algo que melhor... mais potente no grupo. (P2 - CAPS 1) 


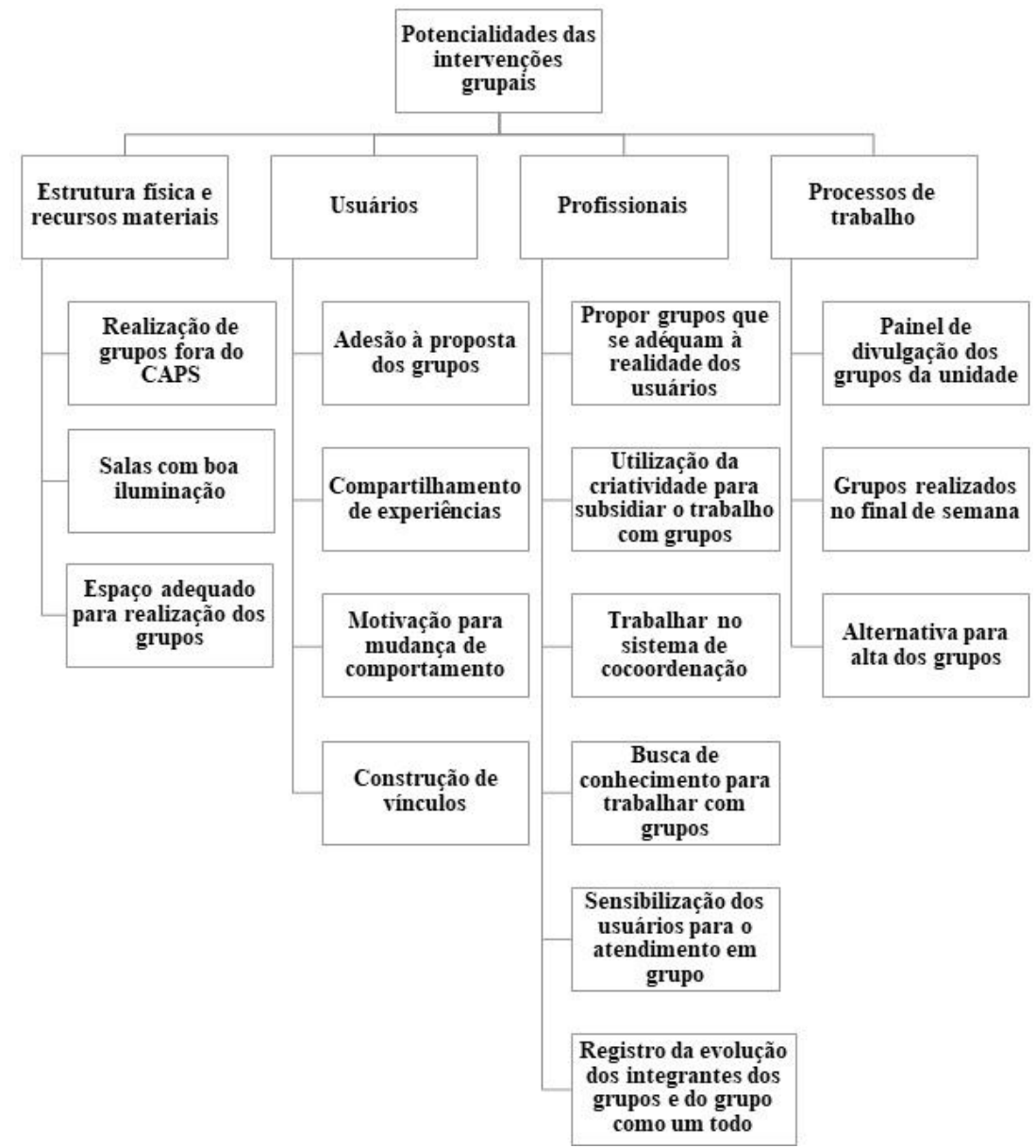

Figura 1. Árvore de decodificação da categoria e das subcategorias do estudo. Elaborado pelos autores. Goiânia/GO, Brasil, 2019.

[...] eu acho que muitos adolescentes que vincularam a unidade através do grupo de videogame e que ficaram em atendimentos individuais ou que foram para outros grupos, eles perderam a concepção de que estavam vindo aqui obrigados, porque o juiz mandou. (P19 - CAPS 3)

Agora tem a caminhada também. [...] Nesse grupo hoje, despertou um sentimento no usuário [...]. Ele lá com a limitação dele, que ele faz uso de tinner, ele foi caminhando [...] e depois voltou e falou: 'Nossa, que efeito bom faz uma caminhada, melhor que o tinner'. (P29 - CAPS 4)

A subcategoria Potencialidades das intervenções grupais relacionadas a aspectos dos profissionais revela as ações realizadas pelos coordenadores de grupos que favorecem uma maior efetividade das intervenções grupais, como a busca de qualificação para trabalharem com grupos:

[...] são muitos profissionais e, assim, muita coisa que transcende até a área de atuação do profissional. O profissional busca outra formação, além da formação básica da profissão, o tempo todo. (P24 - CAPS 4)
A sensibilização dos usuários para os atendimentos grupais e a proposição de grupos que se adequem à realidade dos usuários também foram citadas nos depoimentos dos participantes como potencializadores deste tipo de intervenção:

[...] quando você coloca a proposta do grupo e vai sempre sensibilizando eles. Eu acho que a gente consegue ter uma adesão melhor dos pais. [...] o acolhimento é um ponto importante para sensibilização do grupo de família. (P19 - CAPS 3)

[...] eu tenho percebido que a arte, a música, facilita muito, tanto que quando a gente propõe para os adolescentes o que têm aqui, eles sempre escolhem mais as outras coisas do que um atendimento individual, [...] escolhem coisas que estão mais relacionadas com o universo deles. (P15 - CAPS 3)

Os profissionais também externaram que a utilização da criatividade para subsidiar as atividades grupais que implementam, trabalhar no sistema de cocoordenação com outro colega e 
registrar a evolução dos integrantes dos grupos e do grupo como um todo são fatores que impulsionam tal intervenção:

[...] eu vejo que, para o grupo não morrer, tem que estar sempre você movimentando, criando... a criatividade. Tem que inovar sempre. (P18 - CAPS 3)

Outra coisa que eu acho que ajuda bastante é poder compartilhar isso com outro profissional. Às vezes, o cara te dá uma energia, uma motivação diferente que eu acho que sozinho é bem difícil, porque eu tenho grupos sozinhos e acompanhados. Acho que isso também ajuda. (P17 - CAPS 3)

[...] a gente registra muito mais como que foi aquela pessoa no grupo, o que ela trouxe e aí nesse outro registro, a gente tinha um olhar maior de como que está o grupo, como que as pessoas se relacionaram. (P15 - CAPS 3)

Na subcategoria Potencialidades das intervenções grupais relacionadas aos processos de trabalho, apresentam-se elementos que dizem respeito à cultura organizacional das instituições, que se reflete nos atendimentos grupais, como a criação de intervenções grupais como alternativa de alta dos usuários, realização de grupos em horários alternativos no final de semana e construção de um painel de divulgação das ofertas terapêuticas grupais ofertadas pelo serviço:

Isso, na verdade, não tem um senso comum assim, dessa necessidade de alta, mas, talvez, de pensar grupos para ajudar eles a lidarem com situações fora, de entrar num novo trabalho, das relações [...]. (P7 - CAPS 2)

Mas tem uma coisa que eu acho que diferencia no nosso CAPS, que são os grupos que foram criados no sábado e domingo, porque, como o nosso CAPS é um CAPS 3 , [...] nós temos grupos para usuário, grupos para a família e usuário, temos a horta que também funciona, então, eu acho que esse diferencial tem. (P25 - CAPS 4)

[...] o usuário vem no acolhimento à tarde, mas ele vai frequentar o grupo de manhã, então, a gente tem condições e, se ele está lotado ou se esse grupo se criou um grupo novo de repente, a gente ainda não sabe, tudo isso é colocado nesse painel. (P25 - CAPS 4)

\section{DISCUSSÃO}

No decorrer da investigação e por meio do processo analítico dos dados, foi possível identificar aspectos que evidenciam a potência do trabalho com grupos empreendidos pelos profissionais dos CAPSad ligados à estrutura física e aos recursos materiais dos serviços, bem como aos usuários membros dos grupos, equipes multiprofissionais e processos de trabalho.

Na subcategoria Potencialidades das intervenções grupais relacionadas à estrutura física e recursos materiais, foi explicitada uma estratégia positiva de operacionalização dos grupos fora da instituição e realizada pelos profissionais em função da falta de espaço físico adequado para a realização dessas intervenções nos serviços de origem. Essa ação favoreceu a inclusão dos usuários nos espaços do território, ampliando seus movimentos de reinserção social.

Ainda, considera-se que as atividades grupais são facilitadoras para a ressocialização e inserção social já que promovem, em seu processo de trabalho, o agir e o pensar coletivos, respeitando a diversidade, a subjetividade e a capacidade de cada sujeito ${ }^{4}$. Essas atividades extrapolam a proposta ambulatorial circunscrita ao trabalho médico e farmacológico e que faz contraposição à persistente principalidade da consulta psiquiátrica frente ao repertório de estratégias de suporte psicossocial disponível na atualidade ${ }^{29}$.

Constata-se que a literatura científica internacional expõe limitações ao acesso e à prestação de serviços de saúde em todo o mundo e sinaliza que estratégias com práticas de cuidado em saúde mental territoriais contribuem mais para a inserção e aceitação dos usuários na comunidade ${ }^{30}$. Nessa perspectiva, considera-se o grupo terapêutico como estratégia de produção de cuidado, podendo alterar, favoravelmente, os circuitos de doença-cura e ampliar visões reducionistas dos serviços de saúde do território ${ }^{31}$.

Por outro lado, outros sujeitos deste estudo afirmaram ter espaço adequado no serviço para ofertar atividades grupais, com salas amplas para a realização de grupos e oficinas, sendo que algumas foram planejadas e construídas para acomodar intervenções grupais. Esse fenômeno de adequação do espaço físico não é uma realidade generalizada nos CAPS no Brasil. Evidencia-se que espaços físicos precários contribuem para o surgimento das cargas psíquicas de trabalho por gerar um descontentamento na equipe, refletindo, negativamente, no cuidado psicossocial oferecido aos usuários ${ }^{32}$.

Ressalta-se, ainda, a importância de espaços físicos acolhedores para usuários e trabalhadores nos CAPS porque fortalecem o trabalho e possibilitam o bem-estar aos usuários do serviço. Nota-se que o espaço físico é considerado adequado quando leva em conta a especificidade das pessoas que são atendidas, como ambiente para as diferentes atividades que são realizadas, além de ampliá-las, como, por exemplo, biblioteca e área verde para atividades ao ar livre ${ }^{33}$.

Na subcategoria Potencialidades das intervenções grupais relacionadas aos aspectos dos usuários, os profissionais pesquisados descreveram algumas potencialidades e fenômenos impulsores para as intervenções grupais, tais como o compartilhamento de experiências, a construção de vínculos entre os membros, a adesão às propostas grupais e a conscientização para a mudança de comportamentos que remetem aos fatores terapêuticos do grupo 25 .

Nos serviços públicos de saúde, a assistência por meio de grupos terapêuticos faz parte da realidade cotidiana, pois eles são utilizados como ferramenta para trabalhar a subjetividade dos integrantes dessa modalidade de cuidado ${ }^{34}$. Além disso, 
a intervenção com grupos estimula o trabalho em equipe na perspectiva da multidisciplinaridade com a finalidade de proporcionar um maior bem-estar e qualidade de vida aos usuários ${ }^{35}$.

A riqueza das intervenções grupais em serviços de CAPS é que, além da participação dos usuários no grupo, eles são incentivados a participar na própria gestão do grupo. Ressaltase ainda que esses dispositivos de intervenção contribuem para o fortalecimento do modo de vida dos usuários da saúde mental e de seus familiares ${ }^{36}$. Sabe-se que o grupo impulsiona a reflexão acerca dos sintomas manifestados, dos sofrimentos e do processamento vivido pelo próprio grupo ${ }^{31}$ em razão do vínculo formado entre os participantes que estimula a transformação do sujeito ${ }^{37}$. Percebe-se também o desenvolvimento de aspectos cognitivos considerando-se a interação social como fundamental para o amadurecimento do indivíduo ${ }^{38}$.

Para isso, as interações grupais possibilitam diálogos e troca de experiências valiosas no processo de aprimoramento no modo de viver e socializar, repercutindo na autoimagem e nas relações interpessoais entre os seus membros ${ }^{4}$. Também, permite, aos participantes do grupo, constituírem-se mutuamente como rede de apoio ${ }^{39,40}$.

Em outras palavras, as trocas grupais favorecem a percepção de que os indivíduos não estão sozinhos e/ou que passam por problemas similares aos das outras pessoas, condição que caracteriza o fator terapêutico da universalidade. A catarse outro fator terapêutico grupal, provém da exposição de emoções e sentimentos e da aceitação dos demais. De igual forma, a coesão favorece a aceitação, a compreensão e o sentimento de pertença entre os membros, facilitando que façam revelações pessoais, assumindo riscos ao enfrentar seus medos, receios e dificuldades importantes no processo terapêutico. Outro fator terapêutico importante nos trabalhos grupais é a aprendizagem interpessoal que ocorre a partir das trocas entre os participantes e possibilita a expressão de comportamentos mais efetivos para lidarem com os seus problemas ${ }^{25}$.

Na subcategoria Potencialidades das intervenções grupais relacionadas aos profissionais, os participantes do estudo sinalizaram a preocupação individual de busca de conhecimento sobre tecnologia grupal, além da sua área de formação. Assim, a capacitação e a instrumentalização teóricas para o desenvolvimento de competências para a coordenação de grupos são essenciais para que estes profissionais tenham clareza do processo e da dinâmica dos grupos que estão sob sua responsabilidade e facilitam o aparecimento, a identificação e o reconhecimento dos fatores terapêuticos do grupo ${ }^{9,13}$.

Durante a recepção de novos usuários que chegam no CAPSad para participar da modalidade de tratamento em grupo, o acolhimento foi identificado como uma das potencialidades para o trabalho com grupos no contexto da atenção psicossocial. Isso porque o acolhimento tem como finalidade principal proporcionar, ao usuário que está em sofrimento, ser ouvido em um ambiente sem julgamentos ${ }^{41}$. Além disso, os sentimentos, os desejos e a compreensão do usuário sobre o sofrimento que o traz ao serviço devem ser considerados e sobressair às normas do serviço por meio do respeito à história de vida e à subjetividade de cada indivíduo ${ }^{42}$. Portanto, a escolha pelas ofertas terapêuticas do CAPSad deve ter a participação do próprio usuário.

Nesse sentido, o acolhimento, no CAPS, é um desafio para os profissionais para que sua operacionalização seja concretizada na perspectiva multidisciplinar ${ }^{43}$. Ao sensibilizar e selecionar pessoas para comporem os grupos, o coordenador deve ter clareza dos objetivos e da estrutura grupal e pode basear-se em alguns critérios como a motivação das pessoas para engajarem-se no seu cuidado, problemas que podem ser tratados em uma perspectiva coletiva, o interesse e o compromisso dos indivíduos em comparecer nas sessões, permanecendo até o fim dos encontros ${ }^{25}$. Somada a isso, a seleção de membros para o grupo precisa estar em conformidade com a análise das demandas de cada pessoa ${ }^{14}$.

Outro aspecto relevante do estudo foi o trabalho com sistema de cocoordenação, que potencializou as atividades grupais no cenário da atenção psicossocial, pois, ao atuar em conjunto com outros membros da equipe multiprofissional, os facilitadores sentem-se mais estimulados e motivados durante o seu exercício profissional e conseguem dividir suas impressões sobre o que aconteceu nos atendimentos. Dessa forma, a discussão sobre os fenômenos grupais entre os profissionais da saúde é essencial para que eles consigam fazer a leitura da dinâmica do grupo ${ }^{44}$

Segundo os participantes, a criatividade foi outro recurso potente para o trabalho com grupos no que diz respeito à utilização de práticas criativas durante os atendimentos grupais, além da proposição de ações com vistas a prover recursos e viabilizar o desenvolvimento de suas atividades. Com efeito, os métodos criativos possibilitam ações inovadoras, flexíveis e resolutivas para as demandas do serviço ${ }^{45}$. Em suma, é importante que os profissionais dos serviços de saúde explorem a criatividade como uma ferramenta que os auxilie a cuidar das pessoas que se encontram em sofrimento ${ }^{46}$.

De acordo com o depoimento de um dos profissionais, a oferta de grupos com propostas mais próximas da realidade dos usuários do serviço colabora com o trabalho grupal na atenção psicossocial. Nesse sentido, a escuta e a sensibilidade do coordenador de grupos são recursos importantes para a compreensão dos fenômenos grupais e para o reconhecimento da singularidade de cada integrante que compõe o grupo, valorizando a história de vida de forma a alinhar os objetivos individuais de cada usuário com o (s) objetivo (s) do grupo. Espera-se, portanto, que a realização da atividade/tarefa proposta conte com a participação de todos e concretize-se de forma satisfatória ${ }^{47}$

Por outro lado, as ferramentas de cuidado em saúde mental devem ter ressonância no universo da sociedade ${ }^{48}$. Isso porque uma das estratégias que garantem a adequação do tratamento às necessidades das pessoas é o PTS, o qual também possibilita a avaliação dos resultados advindos da assistência prestada desde que feito regularmente ${ }^{7}$.

Para tanto, a realidade do indivíduo no território deve ser considerada sob a influência de diversos fatores como as relações familiares e sociais, os serviços de saúde e sociais, 
as organizações não governamentais e outras instituições ou serviços presentes onde ele estiver inserido. Deseja-se que estes dispositivos sejam articulados à RAPS, considerando que assim terão mais alcance e efetividade em relação às ações desconexas ou individuais ${ }^{20}$.

Uma outra potencialidade relatada por uma das colaboradoras foi a realização de registros sobre a evolução de cada membro que frequentava os encontros grupais e do desenvolvimento do grupo no decorrer do tempo, mesmo que, no momento da coleta de dados, ela tenha afirmado que não estava realizando essas anotações na frequência como gostaria. É importante que os coordenadores de grupos façam anotações dos encontros grupais, tanto das repercussões das intervenções em cada integrante como da evolução do próprio grupo de forma sistematizada, pois a ausência ou a carência de registros prejudica a avaliação da terapeuticidade ${ }^{9}$.

Na subcategoria Potencialidades das intervenções grupais relacionadas aos processos de trabalho, os depoimentos apontaram que o desenvolvimento de grupos de preparo para a alta dos usuários que estejam mais organizados com as suas questões é um fator positivo, pois se caracteriza como uma estratégia que visa a empoderar as pessoas extramuros do CAPS. Infelizmente, ainda existe uma escassez de pesquisas científicas que tratam da temática de ferramentas de alta para os usuários de CAPS no cenário brasileiro e internacional ${ }^{49}$. Por isso, o término do tratamento em grupo é complexo e as emoções que permeiam esse fenômeno precisam ser trabalhadas: as do sujeito, do coordenador e do grupo. Se a finalização do processo terapêutico em grupo for planejada, o processo de alta pode trazer benefícios para a transformação do indivíduo. Nessa perspectiva, o coordenador deve frisar que a interrupção do tratamento é uma etapa que não precisa ser dolorosa, sugerindo que os demais integrantes colaborem e ajudem o companheiro a encerrar este ciclo ${ }^{25}$.

Outro aspecto importante identificado nas falas dos profissionais foi a disponibilização de grupos terapêuticos aos finais de semana em um CAPSad III, o que aumentou a frequência dos usuários que não conseguem se ausentar do trabalho durante os demais dias da semana nas sessões. Esse dado aponta para a potência deste dispositivo em proporcionar cuidado fora dos horários convencionais, incluindo o acolhimento noturno e o funcionamento em finais de semana e feriados, favorecendo o acesso dos usuários aos CAPS de acordo com suas necessidades e possibilidades. Sabe-se, entretanto, que nem todos os CAPS, no cenário brasileiro, possuem essa configuração e cultura organizacional de atendimento contínuo e, ainda, é um desafio por requerer critério populacional mínimo e quadros de pessoal qualitativa e quantitativamente compatíveis com as demandas de serviços ${ }^{50}$.

Por fim, houve a citação da potencialidade ligada aos processos de trabalho, que consiste na elaboração e na divulgação de um painel na unidade com informações de todos os grupos empreendidos nos períodos matutino e vespertino, facilitando a participação dos usuários. Assim, eles têm acesso a mais informações, podendo optar por grupos que venham também a atender aos seus interesses pessoais, horários e realidade de vida. Tal condição propicia a autonomia dos sujeitos, levando em consideração que a comunicação criativa entre profissionais e usuários dos CAPS sobre o seu tratamento e a forma de dar orientações é uma ferramenta eficaz para promover o protagonismo das pessoas assistidas por esses serviços ${ }^{51}$.

\section{CONSIDERAÇÕES FINAIS E IMPLICAÇÕES PARA A PRÁTICA}

As intervenções grupais nos serviços participantes revelaramse como ferramentas potentes de cuidado e estão devidamente alinhadas às ações esperadas pelos CAPS para a reabilitação e reinserção social dos usuários na comunidade, no fomento de trocas de experiências entre os integrantes do grupo e no estímulo ao enfrentamento para as suas questões existenciais e relacionadas ao uso abusivo de álcool e de outras drogas.

É preciso reconhecer, entretanto, a necessidade premente da educação permanente focada na tecnologia grupal para os trabalhadores dos serviços substitutivos. Cuidar no contexto grupal também pode fortalecer o trabalho em equipe multidisciplinar na medida em que exige ações coletivas, desde o planejamento, a condução e avaliação da proposta terapêutica.

Há a convicção de que o estudo contribui para o campo acadêmico no sentido de sensibilizar os capacitadores para esta temática e no campo assistencial, alertando as categorias profissionais que atuam nos CAPSad, incluindo os enfermeiros (as) e técnicos de Enfermagem, para o valor da atuação com grupos, uma tecnologia leve de cuidado, pois é capaz de oportunizar inúmeros fatores terapêuticos aos usuários.

Há de se considerar algumas limitações do estudo, o qual foi realizado com um público e contexto específicos relacionados a transtornos causados por abuso de substâncias psicoativas, em um cenário que envolveu serviços especializados em saúde mental. Contudo, revelou as potencialidades das intervenções grupais em um circuito de dispositivos CAPS e apontou evidências científicas colaborativas para a atenção psicossocial. Sugere-se, considerando a relevância da aplicabilidade da tecnologia grupal, a realização de outras pesquisas em cenários assistenciais diferentes em que o cuidado seja ofertado em contexto grupal.

\section{AGRADECIMENTOS}

À Fernanda Costa Nunes, Raquel Rosa Mendonça do Vale, Eurides Santos Pinho, Daniel Ribeiro de Almeida e Danielle Xavier Moraes pela colaboração na coleta de dados e à Bárbara Souza Rocha, Lizete Malagoni de Almeida Cavalcante Oliveira, Maria Lúcia Leal e Katarinne Lima Moraes pela contribuição na elaboração de um dos instrumentos de coleta de dados.

\section{CONTRIBUIÇÕES DOS AUTORES}

Desenho do estudo. Johnatan Martins Sousa. Coleta ou produção dos dados. Johnatan Martins Sousa. 
Análise de dados. Johnatan Martins Sousa, Elizabeth Esperidião, Nathália dos Santos Silva, Camila Cardoso Caixeta, Roselma Lucchese.

Interpretação dos resultados. Johnatan Martins Sousa, Elizabeth Esperidião, Marciana Gonçalves Farinha, Camila Cardoso Caixeta, Roselma Lucchese, Nathália dos Santos Silva.

Redação e revisão crítica do manuscrito. Johnatan Martins Sousa, Elizabeth Esperidião, Marciana Gonçalves Farinha, Camila Cardoso Caixeta, Roselma Lucchese, Nathália dos Santos Silva.

Aprovação da versão final do artigo. Johnatan Martins Sousa, Elizabeth Esperidião, Marciana Gonçalves Farinha, Camila Cardoso Caixeta, Roselma Lucchese, Nathália dos Santos Silva.

Responsabilidade por todos os aspectos do conteúdo e a integridade do artigo publicado. Johnatan Martins Sousa, Elizabeth Esperidião, Marciana Gonçalves Farinha, Camila Cardoso Caixeta, Roselma Lucchese, Nathália dos Santos Silva.

\section{EDITOR ASSOCIADO}

Maria Catarina Salvador da Motta (1)

\section{EDITOR CIENTÍFICO}

Ivone Evangelista Cabral (D)

\section{REFERÊNCIAS}

1. Amorim TA, Lucchese R, Silva Na EM, Vera I, Paula NI, Simões ND et al. Determinantes de saúde mental e abuso de substâncias psicoativas associadas ao tabagismo: estudo de caso controle. Cienc Saúde Colet. 2019;24(11):4141-52. http://dx.doi.org/10.1590/1413812320182411.02752018.

2. American Psychiatric Association. M294: manual diagnóstico e estatístico de transtornos mentais DSM-5. Porto Alegre: Artmed; 2014.

3. Gambin KA, Almeida VO, Vitali MM. Psicodrama de grupo e dependência química: trabalhando projetos profissionais e experiências de lazer. Perspectivas em Psicologia. 2020;24(1):1-20. http://dx.doi.org/10.14393/ PPv24n1a2020-57680.

4. Bairos CA. O trabalho do psicólogo em grupos de saúde mental no CAPS. Anu Pesq Ext Unoesc São Miguel do Oeste. 2021;5:e24286.

5. Rézio LA, Fortuna CM, Borges FA. Tips for permanent education in mental health in primary care guided by the Institutional Socio-clinic. Rev Latino-Am Enfermagem. 2019;27:e3204. http://dx.doi.org/10.1590/15188345.3217.3204.

6. Sampaio ML, Bispo Jr JP. Entre o enclausuramento e a desinstitucionalização: a trajetória da saúde mental no Brasil. Trab Educ Saúde. 2021;19:e00313145. http://dx.doi.org/10.1590/1981-7746-sol00313.

7. Silva NS, Sousa JM, Nunes FC, Farinha MG, Bezerra AQ. Challenges in the operationalization of singular therapeutic projects in psychosocial care centers. Psicol Estud. 2020;25:e49996. http://dx.doi.org/10.4025/ psicolestud.v25i0.49996

8. Teixeira PTF. Caps AD: a relevância dos serviços e as contribuições da psicologia. Id on Line Rev. Mult. Psic. 2021;15(54):699-712. http:// dx.doi.org/10.14295/idonline.v15i54.2961.

9. Sousa JM, Vale RRM, Pinho ES, Almeida DR, Nunes FC, Farinha $M G$ et al. Effectiveness of therapeutic groups in psychosocial care: analysis in the light of yalom's therapeutic factors. Rev Bras Enferm. 2020;73(Supl. 1):e20200410. http://dx.doi.org/10.1590/0034-71672020-0410.

10. Andrade JMM, Farinha MG, Esperidião E. Mental Health Nursing: waiting room intervention in integral health care. Rev Bras Enferm. 2020;73(Supl. 1):e20180886. http://dx.doi.org/10.1590/0034-7167-2018-0886.
11. Machado AR, Modena CM, Luz ZMP. Das proposições da política às práticas dos serviços: há novidades nos Centros de Atenção Psicossocial Álcool e Drogas? Physis. 2020;30(1):e300118. http://dx.doi.org/10.1590/ s0103-73312020300118.

12. Junges N, Pereira FP, Castan JU. Grupo de treinamento de habilidades sociais em um Centro de Atenção Psicossocial. Barbarói. 2020;56:24660. http://dx.doi.org/10.17058/barbaroi.v0i0.12258.

13. Nunes FC, Caixeta CC, Pinho ES, Souza ACS, Barbosa MA. Group technology in psychosocial care: a dialogue between action research and permanent health education. Texto Contexto Enferm. 2019;28:e20180161. http://dx.doi.org/10.1590/1980-265x-tce-2018-0161.

14. Nunes FC, Farinha MG, Valentin F, Barbosa MA, Rua MS. Group interventions and action research in health: application possibilities. Millenium. 2020;2(11):65-71. http://dx.doi.org/10.29352/mill0211.07.00273.

15. Ribeiro LS, Rocha DG, Bragé EG, Ramos DB, Vrech LR, Lacchin AJB. Enfrentamento da dependência química na gestação por meio de grupos terapêuticos. Braz J Hea Rev. 2020;3(2):1437-45. http:// dx.doi.org/10.34119/bjhrv3n2-008.

16. Rós IA, Ferreira CAC, Garcia CS. Avaliação da psicoterapia de grupo em pacientes com ansiedade e depressão. Rev Psicol Saúde. 2020;12(1):75-86. http://dx.doi.org/10.20435/pssa.v12i1.830.

17. Picasso R, Silva EA, Arantes DJ. Oficina terapêutica, psicologia e arte: experiência de estágio no Centro de Atenção Psicossocial. Rev NUFEN 2020;12(3):87-102. http://dx.doi.org/10.26823/RevistadoNUFEN.vol12. no03rex.34.

18. Carvalho Júnior ACN, Amparo DM, Nogueira RN. O grupo de escuta como um dispositivo clínico em um Centro de Atenção Psicossocial (CAPS II). Psicol Clin. 2019;31(1):123-43. http://dx.doi.org/10.33208/ PC1980-5438v0031n01A06.

19. Favero CP, Rodrigues J, Silva IP, Santos DVD, Barroso TMMDA, Barbosa SS et al. Grupo de gestão autônoma da medicação num centro de atenção psicossocial: experiência de usuários. Referência. 2019;IV Série(21):91-100. http://dx.doi.org/10.12707/RIV19002.

20. Farinha MG, Silva NS. Saúde mental e projetos intervencionistas em tempos de mudanças de políticas. Rev NUFEN. 2019;11(2):1-2. http:// dx.doi.org/10.26823/RevistadoNUFEN.vol11.n02editorial.

21. Santos NHF, Barbosa SFA, Rodrigues CAO, Araújo DD, Gusmão ROM Vieira MA. Perfil de pacientes atendidos em um Centro de Atenção Psicossocial. Rev Enferm UFPE on line. 2019;13:e242177. https://doi. org/10.5205/1981- 8963.2019.242177.

22. Nascimento TM, Galindo WCM. Grupo operativo em Centros de Atenção Psicossocial na opinião de psicólogas. Pesqui Prát Psicossociais. 2017;12(2):422-38.

23. Rocha ML, Aguiar KF. Pesquisa-intervenção e a produção de novas análises. Psicologia. 2003;3(4):64-73. http://dx.doi.org/10.1590/S141498932003000400010.

24. Souza VRS, Marziale MHP, Silva GTR, Nascimento PL. Translation and validation into Brazilian Portuguese and assessment of the COREQ checklist. Acta Paul Enferm. 2021;34:1-9. https://doi.org/10.37689/ acta-ape/2021ao02631

25. Yalom ID, Leszcz M. Psicoterapia de grupo: teoria e prática. Porto Alegre: Artmed; 2006

26. Afonso MLM, Abade FL. Vamos entrar na roda? a metodologia das rodas de conversa. In: Afonso MLM, Abade FL, editores. Para reinventar as rodas [Internet]. Belo Horizonte: Rede de Cidadania Mateus Afonso Medeiros (RECIMAM); 2008. p. 19-39 [citado 2021 jun 1]. Disponível em: https://ufsj.edu.br/portal-repositorio/File/lapip/ PARA_REINVENTAR_AS_RODAS.pdf

27. Bardin L. Análise de conteúdo. 3. reimp. Lisboa: Edições 70; 2018.

28. Resolução no 466/2012 (BR). Aprova diretrizes e normas regulamentadoras de pesquisas envolvendo seres humanos. Diário Oficial da União [periódico na internet], Brasília (DF), 2012 [citado 2021 jun 1]. Disponível em: https:// bvsms.saude.gov.br/bvs/saudelegis/cns/2013/res0466_12_12_2012. $\mathrm{html}$

29. Lima DKRR, Guimarães J. Articulação da Rede de Atenção Psicossocial e continuidade do cuidado no território: problematizando possíveis relações. Physis. 2019;29(3):e290310. http://dx.doi.org/10.1590/ s0103-73312019290310. 
30. Campos DB, Bezerra IC, Jorge MSB. Produção do cuidado em saúde mental: práticas territoriais na rede psicossocial. Trab Educ Saúde. 2020;18(1):e0023167. http://dx.doi.org/10.1590/1981-7746-sol00231.

31. Costa-Rosa A. O grupo psicoterapêutico no discurso do analista: um novo dispositivo da clínica na atenção psicossocial. Rev Psicol UNESP. 2020;18:55-87.

32. Sousa YA, Oliveira JSA, Chaves AEP, Clementino FS, Araújo MS, Medeiros SM. Psychic burden development related to nursing work in Psychosocial Care Centers. Rev Bras Enferm. 2021;74(Supl. 3):e20200114. http://dx.doi.org/10.1590/0034-7167-2020-0114.

33. Pereira EC, Muller FB, Cordeiro SJ. Os encontros grupais na perspectiva psicanalítica: vivências no CAPS infantil. Terra \& Cult.2020;34(66):10417.

34. Silva CP, Jacob RTS, Freire T, Crenitte PAP, Costa ARA. Grupo terapêutico fonoaudiológico de linguagem: revisão integrativa da literatura. Distúrb Comun. 2021;33(1):114-23. http://dx.doi.org/10.23925/21762724.2021v33i1p114-123.

35. Fernandes ETP, Souza MNL, Rodrigues SM. Práticas de grupo do Núcleo de Apoio à Saúde da Família: perspectiva do usuário. Physis. 2019;29(1):e290115. http://dx.doi.org/10.1590/s0103-73312019290115.

36. Barros AL, Pedrosa JIS. Oficina de planejamento num grupo de Escuta e Acolhimento em Saúde Mental. Rev Enferm UFPI. 2019;8(esp):19-23. http://dx.doi.org/10.26694/2238-7234.8esp19-23.

37. Basso L, Souza RM, Araújo S, Cândido CL. Possibilidade de transformação do sujeito a partir dos vínculos no grupo psicoterapêutico infantil. Vínculo. 2019;16(1):52-68. http://dx.doi.org/10.32467/issn.19821492v16n1p52-68.

38. Dias EB, Melo AMAA, Bonfim CB. Estimulação neuropsicológica com jogos digitais: intervenção em grupo com crianças e adolescentes com rebaixamento cognitivo. Debates em Educação. 2020;12(27):477-98. http://dx.doi.org/10.28998/2175-6600.2020v12n27p477-498.

39. Hayashi EAP, Moriyama JS. Grupo de apoio psicológico a mulheres em situação de infertilidade. Psicologia. 2019;39:e179820. http://dx.doi. org/10.1590/1982-3703003179820.

40. Caldas CC, Guedes MMC, Souza HBF, Oliveira IN, Felisberto MA, Magalhães NMW et al. A importância da equipe multidisciplinar nas oficinas terapêuticas em saúde mental. Rev Cient FAMINAS [Internet] 2019; [citado 2021 jun 1];14(1):53-60. Disponível em: https://www.bing $\mathrm{com} / \mathrm{search?q=A+importância+da+equipe+multidisciplinar+nas+ofici-}$ nas+terapêuticas+em+saúde+mental\&aqs=edge.69i57j69i60.956133j0j4\&pglt=515\&FORM=ANNTA1\&PC $=$ DCTS

41. Almeida AP, Barretto AML, Oliveira DFL, Silva LSP, Rodrigues LC Assistência estudantil psicossocial: caracterização do acolhimento em um projeto de extensão na UFPA. Braz J Develop. 2020;6(3):14402-15. http://dx.doi.org/10.34117/bjdv6n3-347.

42. Marques DFM. Acolhimento em saúde mental: os desafios da escuta Pretextos. 2019;4(7):68-73.

43. Zarpelon MZ, Zambenedetti G. Produção de sentidos acerca do acolhimento entre trabalhadores de um Centro de Atenção Psicossocial. Cad Bras Saúde Ment. 2019;11(29):47-62.

44. Soares SM, Ferraz AF. Grupos operativos de aprendizagem nos serviços de saúde: sistematização de fundamentos e metodologias. Esc Anna Nery Rev Enferm. 2007;11(1):52-7. http://dx.doi.org/10.1590/S141481452007000100007.

45. Revilla E. The creativity dilemma. Rev Adm Empres. 2019;59(2):149-53. http://dx.doi.org/10.1590/s0034-759020190207.

46. Araújo JB, Sá MC. Cenários de restrição e formas de (r) existência no campo da saúde mental: um relato de experiência. Rev Psicol Divers Saúde. 2021;10(1):1-17. http://dx.doi.org/10.17267/2317-3394rpds. v10i1.3303.

47. Cardoso ASF, Mielke FB, Riboldi CO, Soares NV, Olschowsky A, Dall'Agnol CM. Coordenação de grupos na enfermagem: reflexões à luz de Pichon-Rivière. Rev Min Enferm. 2009;13(2):288-92.

48. Lima ICS, Rodrigues PHA, Sampaio JJC. Atenção psicossocial de porta aberta: acesso e desafios da comunicação interprofissional em rede. Essentia. 2019;20(2):37-44. http://dx.doi.org/10.36977/ercct. v20i2.276.

49. Kantorski LP, Guedes AC, Machado RA, Couto MLO, Santos VB Pinheiro GEW. A alta dos usuários no Centro de Atenção Psicossocial. J Nutr Health. 2019;9(3):e199305. http://dx.doi.org/10.15210/jonah. v9i3.15845.

50. Silva MNRMO, Abbad GS, Montezano L. Dinâmica organizacional e o modelo psicossocial de três centros de atenção psicossocial álcool e drogas. Pesqui Prat Psicossociais. 2018;13(2):1-17.

51. Bossato HR, Oliveira RMP, Dutra VFD, Loyola CMD. Nursing and the leading role of the user in the CAPS: a study from the constructionist perspective. Rev Gaúch Enferm. 2021;42:e20200082. 